\title{
The Relationship between Spotted Fever Group Rickettsiae and Ticks in Northeast of Libya
}

\author{
Wedad, S. A. Mohamed, Aisha A. Mohammed, Ahmad M. Ekhnafer, Marfoua S. Ali ${ }^{*}$
}

Department of Zoology, Faculty of Science, Omar Al-Mukhtar University, El -Beida, Libya

DOI: $10.36348 /$ sjbr.2021.v06i02.001 $\quad$ | Received: 09.01.2021 | Accepted: 20.01.2021 | Published: 04.02.2021

*Corresponding author: Marfoua S. Ali

\section{Abstract}

Spotted fever group Rickettsiae are predominantly transmitted by ticks. Rickettsiae have developed many strategies to adapt to different environmental conditions, including those within their arthropod vectors and vertebrate hosts. The tickRickettsiae relationship has been a point of interest for many researchers, with most studies concentrating on the role of ticks as vectors. Unfortunately, less attention has been directed towards the relationship of Rickettsiae with tick cells, tissues, and organs. This work was carried out to identify and prepare smear from salivary gland of ticks. Ticks were collected from June to September, 2018 in vegetation around residences $(n=30)$ of people who have been in hospital as a result of symptoms related to spotted fever. Some samples were collected from patients who diagnosed with symptoms related to spotted fever in El-Beida City. Ticks were sorted by species and life stage into pools for analyses for pathogens. Smear of salivary gland for male and female of thick were stained with gram negative bacteria and examined under microscope. Results from stains were positive for the presence of bacteria in female sample and negative for male sample.

Keywords: Spott fever, patients, ticks, El-Baida City, Libya.

Copyright (C) 2021 The Author(s): This is an open-access article distributed under the terms of the Creative Commons Attribution 4.0 International License (CC BY-NC 4.0) which permits unrestricted use, distribution, and reproduction in any medium for non-commercial use provided the original author and source are credited.

\section{INTRODUCTION}

Rocky Mountain spotted fever (RMSF), also known as blue disease, is the most lethal and most frequently reported Rickettsial illness in many centuries. RMSF was first identified in the Rocky Mountain region of the United States and the condition is now recognized as broadly distributed throughout the contiguous states [1]. Mountain Spotted Fever (MSF) is endemic to the Mediterranean area, including northern Africa and southern Europe, some cases was described in Algeria, Malta, Cyprus, Slovenia, Croatia, Kenya, Somalia, South Africa, and in areas surrounding the Black Sea. Spotted fever cases have been confirmed as MSF by the use of molecular tools in Portugal, Italy, Malta, Greece, Croatia, Spain, France and South Africa $[2,3]$. Rickettsia conorii is raelensis has been described in Tunisia, Libya, Sardinia-Italy, and Portugal [4]. Initial signs and symptoms of the disease include sudden onset of fever, headache, and muscle pain, followed by development of rash. The disease can be difficult to diagnose in the early stages, and without prompt and appropriate treatment it can be fatal [5]. Tick-borne rickettsioses are an important cause of human morbidity worldwide [6]. It is distinct from the viral tick-borne infection. The disease is caused by Rickettsia rickettsii, a species of bacterium that is spread to humans by Dermacentor ticks [7]. It is a zoonotic disease that is maintained in nature in a cycle involving a tick vector and reservoir, with various wildlife species contributing to new lines of ticks infected with the pathogen [8] Humans are incidentally infected. The epidemiology of each tick-borne Rickettsial disease reflects the geographic distribution and seasonal activities of the tick vectors and vertebrate hosts involved in the transmission of these pathogens, as well as the human behaviors that place persons at risk for tick exposure, tick attachment, and subsequent infection $[9,10]$. Ticks transmit $\mathrm{R}$ rickettsii to a vertebrate principally during their feeding behavior. However, human infection has occurred much less often following transmural or inhalation exposure to tick fluids (hemolymph), tick feces, or crushed tick tissues. In the natural history of $\mathrm{R}$ rickettsii transmission, human and domestic dog infections are considered incidental events. $R$. ricketti resides principally in various species of ticks, which are considered the reservoirs or natural hosts [11]. In the United States, the tick species that are most frequently associated with transmission of $\mathrm{R}$. rickettsii is the 
American dog tick, Dermacentor variabilis (Figure 1 A), the Rocky Mountain wood tick, Dermacentor andersoni (Figure $1 \mathrm{~B}$ ), the brown dog tick, Rhipicephalus sanguineus ((Figure $1 \mathrm{C}$ ), which is located throughout the United States. This species was recorded in Libya in Giado, Sirte, Misurata, Tigi, Bir El Raim, Zliten, Gardinia, Benina, Wadi Ahmar, Bengazi, Ghemines, Derna and Sebha [12, 13] . Rhipicephalus sanguineus was recorded in farms surrounding El-Baida city in Al-Jabal Al-Akhder [14], the Gulf Coast tick, Amblyomma maculatum (Figure $1 \mathrm{D}$ ), the lone star tick, Amblyomma americanum (Figure $1 \mathrm{E}$ ), The blacklegged tick, Ixodes scapularis (Figure 1 F) [15]. Several tick species of the genus Amblyomma are vectors of $\mathrm{R}$. rickettsii from Mexico to Argentina, including A. cajennense, A. aureolatum, A. imitator, and A. sculptum $[16,17]$.

Due to presence of tick [14], Rickettsia, symptoms related to spotted fever during seasonal activities of the tick and increasing number of cases diagnosed with spotted fever [5]. This work was attempted to gain a better understanding of whether species of Rickettsia are present in region of study, with the main emphasis on the tick-borne spotted fever group. Therefore, samples of ticks were collected to prepared smear from their salivary gland to confirm presence of Rickettsia.
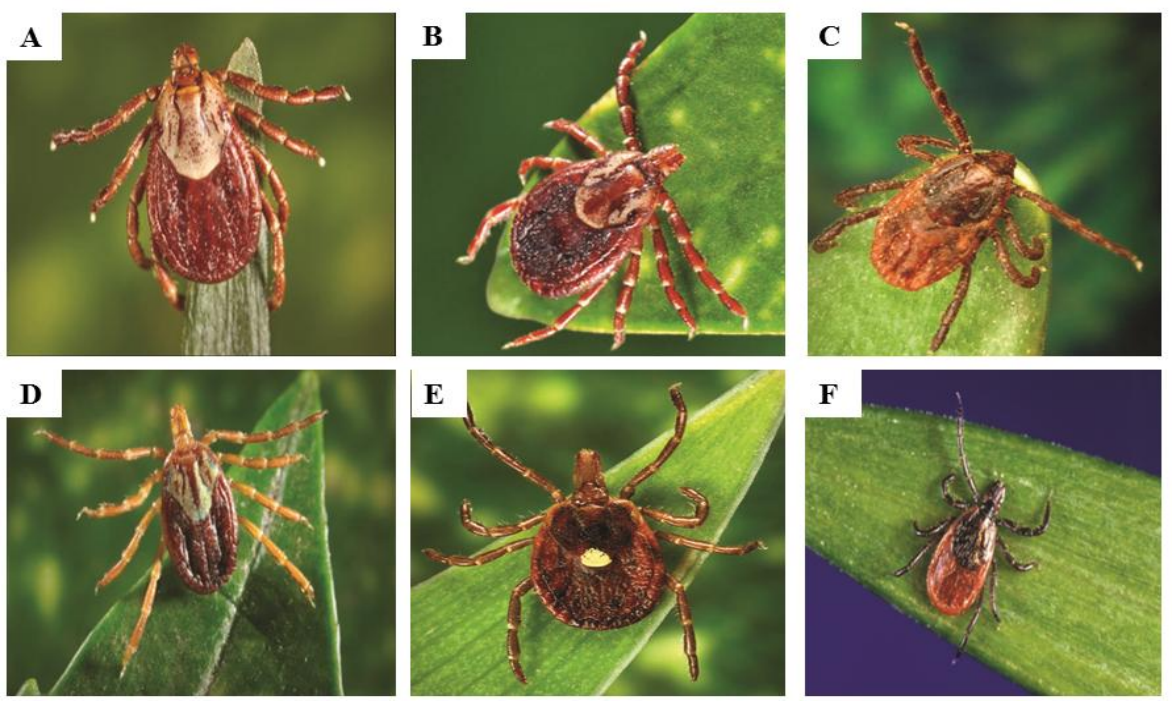

Fig-1: The main tick species involved in transmission of RMSF, The American dog tick, Dermacentor variabilis (A), the Rocky Mountain wood tick, Dermacentor andersoni (B), the Gulf Coast tick, Amblyomma maculatum (D), the lone star tick, Amblyomma americanum (E) and the blacklegged tick, Ixodes scapularis (F) [15]

\section{MATERIALS AND METHODS}

The study protocol was reviewed and approved by Bioethics Committee at Biotechnology Research Center (BEC-BTRC) with Ref No: BECBTRC 07-2018. Inclusion criteria involve: patients agreement participation in the study

Patients experiencing flu-like symptoms of MSF after confirmed or suspected tick bite were included in the study. Patients were seeking medical attention from May to October 2018 at Al-Thowra Hospital in El-Beida City. Data on the patient demographic characteristics, including their area of residence, and proximity to animals were gathered from patients. Ticks were collected from June to September, 2018 in vegetation around residences $(n=30)$ of people who have been in hospital as a result of symptoms related to spotted fever. Sample of ticks were collect at residence that confirm with at least two cases. Subsequently, ticks were sorted by species and life stage into pools for analyses for pathogens.

Any tick collected was labeled with a unique consecutive identifier or non-engorged when removed from the canine and the stage of the tick was also noted, as nymph or adult, identified using several keys and a dissecting microscope and then stored in $70 \%$ ethanol until processed. Ticks were identified based on their morphology by light microscopy, using a dissecting microscope and the taxonomic key outlined in [18]. Where possible, the species were identified. However, in some cases, mouthparts or other areas of the body used for differentiation were damaged during removal from the host and it was only possible to identify them to the genus level $[19,20]$.

Each tick was split into two parts (anterior and posterior) at the area between coxa 1 and coxa 2 . The anterior part was then removed from the posterior part using sterile forceps. Since the salivary glands were attached with the anterior part, they could be removed from the tick carcass without damaging the midgut. Ticks were placed ventral side up; and by carefully pushing the fine needle of a $50 \mu$ l syringe beneath the integument and toward the anterial part of the tick, the solution was inserted into the tick. Salivary secretions were collected within five minutes. The salivary glands were then collected into a sterile $1.5 \mathrm{ml}$ tube using 
sterile forceps, followed by washing with sterile phosphate-buffered saline (PBS) in order to minimize bacterial contamination [21]. To determine whether ticks were infected by the agent of Rickettsia, we examined their salivary glands. The tick saliva of both sexes was tested. Those examined for salivation was glued on pasting-tape, fixed to a glass slide, and inoculated in the hind part of the all oscutum with up to $0.3 \mathrm{ml}$ of $\mathrm{I} \%$ pilocarpine, the amount depends on the period of engorgement. The secreted saliva was collected from the top of the hypostome and chelicerae of each tick by mean sofa very fine capillary and was either transferred to a glass slide for the preparation of a smear. The coxiellae were again stained on the smears by the Method of Gimenez [22].

Tick salivary glands were excised into PBS $10 \%$ fetal bovine serum and were allowed to dry on gelatin-coated slides. These preparations were fixed in absolute methanol for $10 \mathrm{~min}$, hydrolyzed in $1 \mathrm{MHCl}$ at $60^{\circ} \mathrm{C}$ for $12 \mathrm{~min}$, immersed in Schiffsre agent (Sigma) for $1.5 \mathrm{hr}$, counter stained in alcoholic fastgreen $(0.1 \%)$, dehydrated, clearedinxylene, and mounted in Permount. The slides were examined under bright field illumination at $\mathrm{x} 400$ for the presence of pink (DNAcontaining) inclusions between the salivary acinar nuclei [22].

\section{RESULTS}

This study was carried out in the 7 consecutive months (May-November 2018). 129 was the total number of patients who were diagnosed with symptoms related to spotted fever. The number of patients for previous four years was ranged from 90-135 each year (data from statistical department in hospital). Initial symptoms include sudden onset of fever, headache, chills, malaise, and myalgia. Other early symptoms might include nausea or vomiting, abdominal pain, anorexia, and photophobia. A rash typically appears 24 days after the onset of fever (Figure-2).
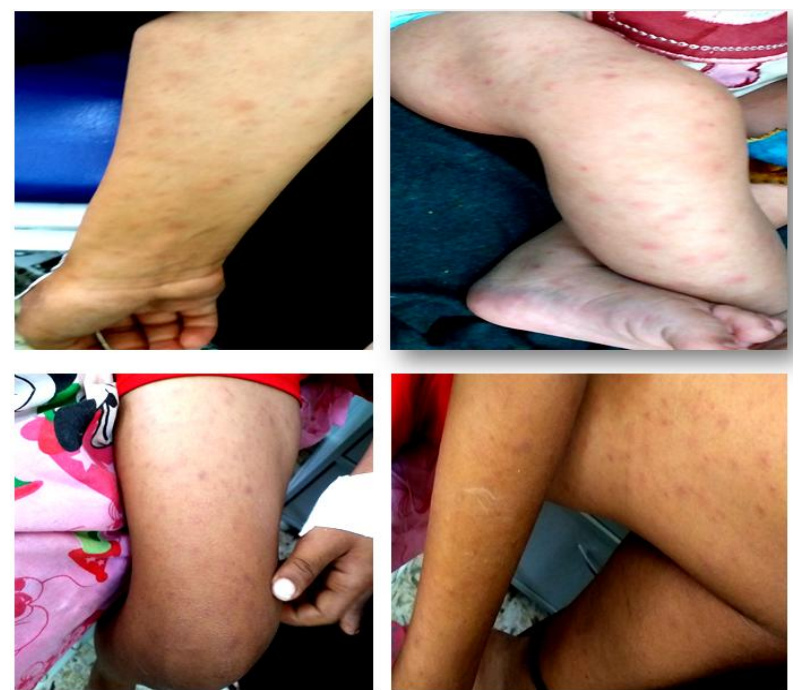

Fig-2: A rash typically appears 2-4 days after the onset of fever in some cases
Most common ticks can be identified microscopically to the species level by examining mouthparts, scutum, accessory adanal plates, anal groove, capitulum shape, presence or absence of eyes, festoon, central festoon, coxae type, genital pore, hypostomal teeth, lateral grooves and leg coloration.

Ticks were collected from Akfenta, Alwseta, Qaser Libya, Sede Abdalwahed, Omer Almukhtar where the cases of spotted fever (male and female) identified (Figure 3 (A-D)). Salivary glands from male and female ticks were collected (Figure 4 (A-D)). Smears of slivery glands stained for gram negative bacteria and examined under microscope. These stains for positive for the presence of bacteria in female samples as shown in Figure 5 and negative for male samples as shown in Figure 6.
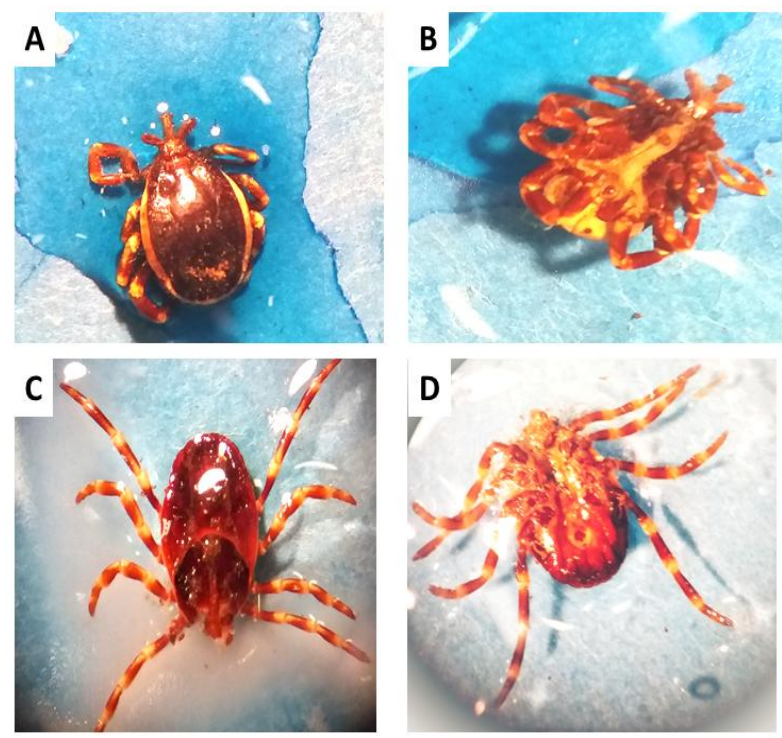

Fig-3: Dorsal (A) and ventral (B) views of male ticks. Dorsal (C) and ventral (D) views of female (C and D) ticks
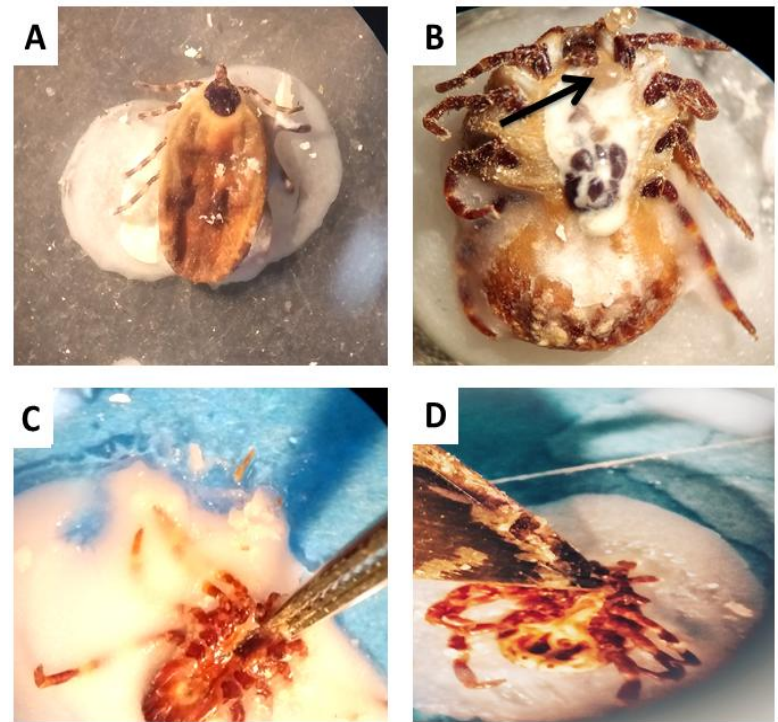

Fig-4: Steps to isolate salivary gland of tick (Black arrow) 


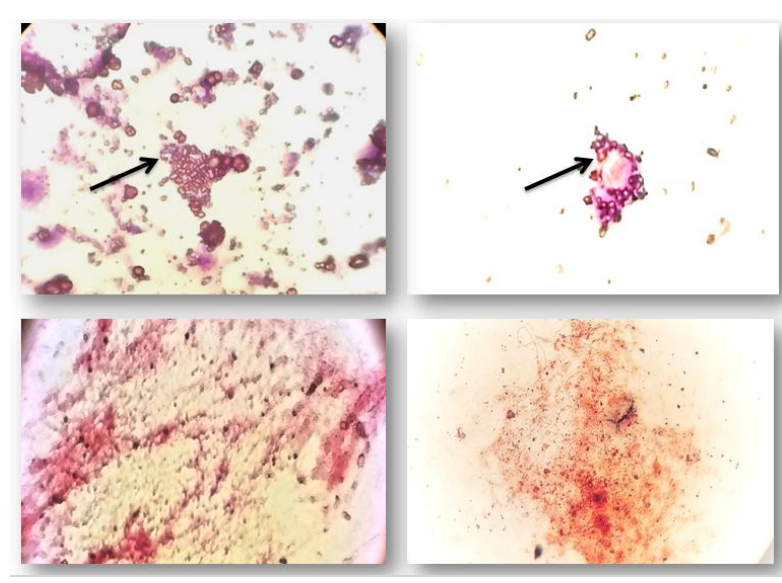

Fig-5: Smear from salivary gland (Female tick) that stain with gram positive bacteria

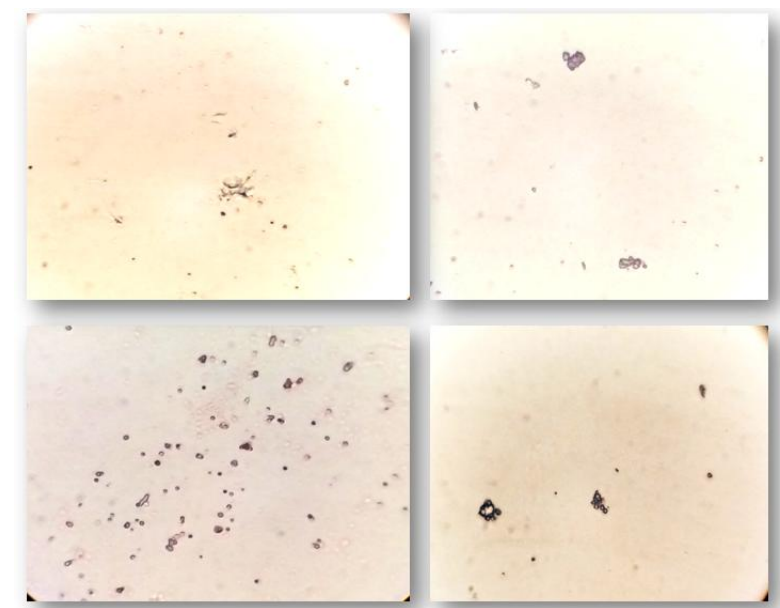

Fig-61: Smear from salivary gland (male tick) that stain with gram negative bacteria

\section{DISCUSSION}

The epidemiologic and clinical characteristics of the patients described in this study were strongly compatible with spotted fever. All patients resided in areas where ticks were present, most of them were resided in farms, others had been contact with animals at some point or had picnic in open area. All patients became ill in mid-spring (May) to early an autumn (November) (2018), and presented with symptoms characterized by myalgias, vomiting, and/or rash. The clinical symptoms and signs were similar to ones presented in other studies [23-26]. Geographical distribution for all subjects in our study was from twenty different locations around El-Beida City. These locations were farms and places where animals are found (sheep, cows and dogs) and presence of ticks was very common. The monthly distribution of cases shows that the apparition of the disease parallels the maximal activity of the immature stages of tick (MayNovember). The first study on tick-Rickettsiae interactions was carried out by Ricketts during his investigation of RMSF in western Montana, in which he demonstrated that $\mathrm{D}$. andersoni was the principal vector of R. rickettsii [27]. Subsequently, naturally infected ticks were used to investigate such interactions, including the vertical transmission of $\mathrm{R}$. conorii in $\mathrm{Rh}$. sanguineus [28], of R. africae in A. hebraeum [29] and of R. massiliae in Rh. turanicus [30]. They were also used to study the impact of Rickettsiae on their host's physiology and reproduction [31]. One of the challenges of using wildcaught ticks is the collection of sufficient numbers of infected ticks (because the prevalence of infection in nature may be low) and their maintenance in a laboratory environment.

In conclusion, spotted fever is an overall benign disease. Continuing investigations on tick-Rickettsiae interactions may provide a better understanding of the factors influencing the emergence and distribution of arthropod transmitted pathogens and their co-evolution

\section{REFERENCES}

1. Chapman, A. S. (2006). Diagnosis and management of tickborne rickettsial diseases; Rocky Mountain spotted fever, ehrlichioses, and anaplasmosis--United States: a practical guide for physicians and other health-care and public health professional. 55(RR-4):1-27.

2. Buczek, W., Koman-Iżko, A., Buczek, A. M., Buczek, A., Bartosik, K., Kulina, D., \& Ciura, D. (2020). Spotted fever group rickettsiae transmitted by Dermacentor ticks and determinants of their spread in Europe. Annals of Agricultural and Environmental Medicine.

3. Rovery, C., Brouqui, P., \& Raoult, D. (2008). Questions on Mediterranean spotted fever a century after its discovery. Emerging infectious diseases, 14(9), 1360-1367.

4. Parola, P., Paddock, C. D., Socolovschi, C., Labruna, M. B., Mediannikov, O., Kernif, T., ... \& Raoult, D. (2013). Update on tick-borne rickettsioses around the world: a geographic approach. Clinical microbiology reviews, 26(4), 657-702.

5. Alexandrov, E., Mitov, D., Kamarintchev, B., \& Bogdanov, N. (1999). Current features of Mediterranean spotted fever in Bulgaria in contemporary conditions. Rickettsiae and rickettsial diseases at the turn of the third millennium. Paris: Elsevier, 279-81.

6. Parola, P., Paddock, C. D., \& Raoult, D. (2005). Tick-borne rickettsioses around the world: emerging diseases challenging old concepts. Clinical microbiology reviews, 18(4), 719-756.

7. Chapman, A. S., Murphy, S. M., Demma, L. J., Holman, R. C., Curns, A. T., McQuiston, J. H., ... \& Swerdlow, D. L. (2006). Rocky Mountain spotted fever in the United States, 19972002. Vector-Borne \& Zoonotic Diseases, 6(2), 170-178.

8. Parola, P., \& Raoult, D. (2001). Ticks and tickborne bacterial diseases in humans: an emerging infectious threat. Clinical infectious diseases, 32(6), 897-928. 
9. Nicholson, W. L., Paddock, C. D., Demma, L., Traeger, M., Johnson, B., Dickson, J., ... \& Swerdlow, D. (2006). Rocky Mountain spotted fever in Arizona: documentation of heavy environmental infestations of Rhipicephalus sanguineus at an endemic site. Annals of the New York Academy of Sciences, 1078(1), 338-341.

10. Openshaw, J. J., Swerdlow, D. L., Krebs, J. W., Holman, R. C., Mandel, E., Harvey, A., ... \& McQuiston, J. H. (2010). Rocky Mountain spotted fever in the United States, 2000-2007: interpreting contemporary increases in incidence. The American journal of tropical medicine and hygiene, 83(1), 174-182.

11. Biggs, H. M., Behravesh, C. B., Bradley, K. K., Dahlgren, F. S., Drexler, N. A., Dumler, J. S., ... \& Traeger, M. S. (2016). Diagnosis and management of tickborne rickettsial diseases: Rocky Mountain spotted fever and other spotted fever group rickettsioses, ehrlichioses, and anaplasmosisUnited States: A practical guide for health care and public health professionals. Morbidity and Mortality Weekly Report: Recommendations and Reports, 65(2), 1-44.

12. Gabaj, M. M., Awan, M. A. Q., \& Beesley, W. N. (1992). A survey of ticks on farm animals in Libya. Annals of Tropical Medicine \& Parasitology, 86(5), 543-548.

13. Hoogstraal, H., \& Kaiser, M. N. (1960). Observations on ticks (Ixodoidea) of Libya. Annals of the Entomological Society of America, 53(4), 445-457.

14. Hador, A. B. (2015). Survey on Ticks in Some Surrounding Farms of Al-Baida, Al-Jabel AlAkhdar, Libya, in Department of Zoology 2015, University of Omar Al-Mukhtar: Al-Baida. Libya.

15. Billings, A. N., Teltow, G. J., Weaver, S. C., \& Walker, D. H. (1998). Molecular characterization of a novel Rickettsia species from Ixodes scapularis in Texas. Emerging infectious diseases, 4(2), 305.

16. Oliveira, K. A., Pinter, A., Medina-Sanchez, A., Boppana, V. D., Wikel, S. K., Saito, T. B., ... \& Bouyer, D. H. (2010). Amblyomma imitator ticks as vectors of Rickettsia rickettsii, Mexico. Emerging infectious diseases, 16(8), 1282.

17. Szabó, M. P. J., Pinter, A., \& Labruna, M. B. (2013). Ecology, biology and distribution of spotted-fever tick vectors in Brazil. Frontiers in cellular and infection microbiology, 3(27):9.

18. Walker, A. (2003). Ticks of domestic animals in Africa: a guide to identification of species, Edinburgh: Bioscience Reports.

19. Furman, D. P., \& Loomis, E. C. (1984). The ticks of California (Acari: Ixodida). Vol. 25. Berkeley-
Losangeles-London: University of California Press.

20. Misurelli, J. (2010). Guidelines for tick identification. Wisconsin Clinical Laboratory Network Laboratory Technical Advisory Group, 1-14.

21. Qiu, Y., Nakao, R., Ohnuma, A., Kawamori, F., \& Sugimoto, C. (2014). Microbial population analysis of the salivary glands of ticks; a possible strategy for the surveillance of bacterial pathogens. PloS one, 9(8), e103961.

22. Rehácek, J., \& Brezina, R. (1968). Detection of Coxiella burneti in saliva of experimentally infected ticks, Hyalomma dromedarii Koch. Bulletin of the World Health Organization, 39(6), 974.

23. Colomba, C., Saporito, L., Polara, V. F., Rubino, R., \& Titone, L. (2006). Mediterranean spotted fever: clinical and laboratory characteristics of 415 Sicilian children. BMC infectious diseases, 6(1), 60 .

24. Germanakis, A., Psaroulaki, A., Gikas, A., \& Tselentis, Y. (2006). Mediterranean spotted fever in Crete, Greece: clinical and therapeutic data of 15 consecutive patients. Annals of the New York Academy of Sciences, 1078(1), 263-269.

25. Kuloglu, F., Rolain, J. M., Akata, F., Eroglu, C., Celik, A. D., \& Parola, P. (2012). Mediterranean spotted fever in the Trakya region of Turkey. Ticks and tick-borne diseases, 3(5-6), 298-304.

26. Pitigoi, D., Olaru, I. D., Badescu, D., Rafila, A., Arama, V., \& Hristea, A. (2013). Mediterranean spotted fever in southeastern Romania. BioMed research international, 2013; 4.

27. Raoult, D., Kohler, J. L., Gallais, H., De Micco, P., Rousseau, S., \& Casanova, P. (1982). Fatal rickettsiosis. La Nouvelle presse medicale, 11(8), 607.

28. Mouffok, N., Benabdellah, A., Richet, H., Rolain, J. M., Razik, F., Belamadani, D., ... \& Raoult, D. (2006). Reemergence of rickettsiosis in Oran, Algeria. Annals of the New York Academy of Sciences, 1078(1), 180-184.

29. Kelly, P. J., \& Mason, P. R. (1991). Transmission of a spotted fever group rickettsia by Amblyomma hebraeum (Acari: Ixodidae). Journal of medical entomology, 28(5), 598-600.

30. Matsumoto, K., Ogawa, M., Brouqui, P., Raoult, D., \& Parola, P. (2005). Transmission of Rickettsia massiliae in the tick, Rhipicephalus turanicus. Medical and veterinary entomology, 19(3), 263-270.

31. Zhong, J., Jasinskas, A., \& Barbour, A. G. (2007). Antibiotic treatment of the tick vector Amblyomma americanum reduced reproductive fitness. PloS one, 2(5), e405. 